\title{
ON THE IMAGE OF SYMPLECTIC COBORDISM IN UNORIENTED COBORDISM
}

\author{
FRED W. ROUSH
}

\begin{abstract}
It is shown in this paper that all 16th powers of unoriented cobordism classes can be represented by stably almost symplectic manifolds. The generators are coefficients in the expansion of a Conner-Floyd characteristic class of the symplectification of a tensor product of symplectic line bundles.
\end{abstract}

In this paper it will be shown that all 16th powers of unoriented cobordism classes are in the image of symplectic cobordism. The method will be similar to those of Ray [4]. Some alternative, more geometric constructions will be given without proof, because the computations involved are considerably longer.

Previously Stong [6] proved that in dimensions 20 and lower this image is zero, Segal [5] found that the first nonzero class in the image is $\left(R P^{2}\right)^{16}$, and Floyd [2] showed that the image must lie in the ring generated by certain 8th and 16th powers.

In Ray's methods one considers a pair of spaces for which the spectral sequence from ordinary cohomology to symplectic cobordism collapses, so that they are "free" (allowing infinite sums) over the symplectic cobordism of a point. Bases are found for the symplectic cobordism of the two spaces, frequently involving the characteristic classes of Conner and Floyd [1, Corollary 8.2]. A map is chosen from one space to the other, and the image of one basis is expanded in terms of the other. The coefficients are the desired classes in the symplectic cobordism of a point.

Here we will consider the space $H P^{\infty} \times H P^{\infty}$. Let $K$ and $K^{\prime}$ denote the canonical symplectic line bundles over the two quaternionic projective space factors, and let $E_{1}(K)$ and $E_{1}\left(K^{\prime}\right)$ denote their Conner-Floyd characteristic classes in $\Omega_{\mathrm{Sp}}^{4}\left(H P^{\infty} \times H P^{\infty}\right)$.

Then $E_{1}(K)^{i} E_{1}\left(K^{\prime}\right)^{j}$ is a basis in the sense mentioned above, for

Received by the editors August 17, 1972.

AMS (MOS) subject classifications (1970). Primary 57D90; Secondary 57D75.

Key words and phrases. Symplectic cobordism, unoriented cobordism, characteristic numbers.

(c) American Mathematical Society 1973 
$\Omega_{\mathrm{Sp}}^{*}\left(H P^{\infty} \times H P^{\infty}\right)$. This can be proved by standard spectral sequence arguments, or by ones similar to that on pp. 50-51 of Conner and Floyd [1] for $H P^{n}$, then taking inverse limits.

There is also the real vector bundle $K K^{\prime}$ over this space, which is the symplectic tensor product of $K$ and $K^{\prime}$ and has real dimension 4. Also its symplectification, $e_{\mathrm{Sp}}\left(K K^{\prime}\right)$, of symplectic dimension 4 . We will consider its Conner-Floyd characteristic class $E_{4}$ in $\Omega_{\mathrm{Sp}}^{16}\left(H P^{\infty} \times H P^{\infty}\right)$.

THEOREM. If $E_{4}\left(e_{\mathrm{Sp}}\left(K K^{\prime}\right)\right)$ is expanded as a sum $\sum m_{i j} E_{1}(K)^{i} E_{1}\left(K^{\prime}\right)^{j}$ with the $m_{i j}$ in the symplectic cobordism of a point, the image of the $m_{i j}$ in unoriented cobordism generates the subring of all 16th powers.

I. Characteristic classes to ordinary cohomology. First we will describe the characteristic classes from symplectic vector bundles to ordinary cohomology. We will use the $s_{\omega}$ symplectic Pontryagin classes in a total class notation which is said to have first appeared in a paper of Boardman. If $\omega=\left(i_{1}, i_{2}, \cdots, i_{n}\right)$, the $s_{\omega}$ class on a sum of line bundles $x_{1}+x_{2}+\cdots+x_{m}$ is the sum of $p_{1}^{S}\left(x_{1}\right)^{i_{1}} p_{1}^{S}\left(x_{2}\right)^{i_{2}} \cdots p_{1}^{S}\left(x_{n}\right)^{i_{n}}$ and all the similar but distinct terms that can be obtained from this one by replacing $x_{1}$ through $x_{n}$ with a different set of $n x$ 's chosen from the $m x$ 's. For each $\omega$ this defines a unique characteristic class.

The corresponding total class is the formal sum $\sum s_{\omega} t_{1}^{a_{1}} t_{2}^{a_{2}} \cdots$, where if $\omega=\left(i_{1}, i_{2}, \cdots, i_{n}\right), a_{1}$ is the number of 1 's among the $i_{k}, a_{2}$ is the number of 2's among the $i_{k}$, and so on. This sum will be denoted $s_{t}$ or $s_{t}(v)$ for a symplectic vector bundle $v$.

It has the properties

(1) $s_{t}(x)=1+t_{1} p_{1}^{S}(x)+t_{2}\left(p_{1}^{S}(x)\right)^{2}+\cdots$

for a symplectic line bundle $x$, and

(2) $s_{t}(v+w)=s_{t}(v) s_{t}(w)$.

From this functor on vector bundles we obtain a related functor from symplectic cobordism to the power series ring in the $t$ 's with coefficients in ordinary cohomology. For the class of the identity map $M$ Sp to itself, this is the image of $s_{t}$ of the canonical bundle under the Thom isomorphism. For a symplectic cobordism class represented by a map from $X$ to $M$ Sp the image of the class for $1_{M S p}$ is taken to be the value of the functor. It will be denoted $S_{t}$, or $S_{t}(a)$ for a symplectic cobordism class $a$.

$S_{t}$ has the properties:

(1) $S_{t}\left(E_{1}(x)\right)=p_{1}^{S}(x)+t_{1}\left(p_{1}^{S}(x)\right)^{2}+t_{2}\left(p_{1}^{S}(x)\right)^{3}+\cdots$,

(2) $S_{t}(a b)=S_{t}(a) S_{t}(b)$,

(3) $S_{t}(a+b)=S_{t}(a)+S_{t}(b)$.

The third of these corresponds to the fact that suspended characteristic classes are additive. 
The first results from the first above and the representation of $E_{1}(K)$ as the composition $H P^{\infty}=M \mathrm{Sp}(1) \rightarrow M \mathrm{Sp}$, with $p_{1}^{S}(K)$ being the Thom class, for the universal example.

Another property of $S_{t}$ that will be needed is its relation with the usual characteristic numbers of manifolds, in the cobordism of a point. Let $S_{\omega}$ denote the coefficient of the same monomial in the $t$ 's in $S_{t}$ that $s_{\omega}$ is in $s_{t}$.

Then $S_{\omega}$ is represented by the image of $s_{\omega} U$ under the composition $S^{k} \rightarrow T \nu(M) \rightarrow M$ Sp which represents the bordism class. Here $U$ is the Thom class for $M \mathrm{Sp}, v(M)$ is the normal bundle of some embedding of a manifold $M$, and $T \nu(M)$ is its Thom space. The first map is an isomorphism on $H^{k}$; the second sends $s_{\omega} U$ to $s_{\omega}(\nu(M)) U_{1}$, where $U_{1}$ is the Thom class of $T \nu(M)$ induced from $U$. The conclusion is that the $S_{t}$ functor on this class is the characteristic number which is the evaluation of the $s_{t}$ class of the normal bundle of $M$, at least $\bmod 2$.

It follows from property (2) that this is the evaluation of $s_{t}^{-1}$ of the tangent bundle of $M$.

A similar construction can be made with symplectic Pontryagin classes replaced by Stiefel-Whitney classes. This will be denoted by $S_{t 0}$ on cobordism classes and $s_{t 0}$ on vector bundles.

The relation of these two classes is that $S_{t 0}$ and $s_{t 0}$ are $S_{t}$ and $s_{t}$ with each $t_{n}$ replaced by $t_{n}^{4}$, and the coefficients reduced mod 2. It is sufficient to prove this for the case of $s_{t 0}$ of a line bundle, by splitting and the properties mentioned above. From the result on pp. 74-75 of Stong [7], $p_{1}^{S}=c_{2}=w_{4} \bmod 2$. From this result it follows that the restriction from the mod 2 cohomology of $H P^{\infty}$ to that of $R P^{\infty}$ induced by a map representing the symplectification of the canonical real line bundle will be monic on mod 2 cohomology, sending $p_{1}^{S}$ to $w_{4}$ of this symplectification, which will be $w_{1}^{4}$ if $w_{1}$ denotes the Stiefel-Whitney class of the canonical real line bundle over $R P^{\infty}$.

Therefore the restriction of $s_{t} \bmod 2$ will be $1+w_{1}^{4} t_{1}+w_{1}^{8} t_{2}+\cdots$. The restriction of $s_{t 0}$ is $s_{t 0}$ of the restricted bundle, which as a real vector bundle is 4 times the canonical real line bundle. So it will be

$$
\left(1+w_{1} t_{1}+w_{1}^{2} t_{2}+\cdots\right)^{4}=1+w_{1}^{4} t_{1}^{4}+w_{1}^{8} t_{2}^{4}+\cdots .
$$

This proves the relation of the two characteristic classes.

II. Proof of theorem. From the introductory section we have an equation $E_{4}\left(e_{\mathrm{Sp}}\left(K K^{\prime}\right)\right)=\sum m_{i j} E_{1}(K)^{i} E_{1}\left(K^{\prime}\right)^{j}$. In order to study the $m_{i j}$, first we will restrict this equation to the symplectic cobordism of $R P^{\infty} \times$ $R P^{\infty}$ and then take $S_{t}$ classes of both sides.

The restriction of $K K^{\prime}$ will be the real vector bundle $4 x x^{\prime}$, where $x$ and 
$x^{\prime}$ denote the canonical real line bundles over the factors; it can be noted that the restrictions of $K$ and $K^{\prime}$ are $e_{\mathrm{Sp}}(x)$ and $e_{\mathrm{Sp}}\left(x^{\prime}\right)$ respectively.

$$
\begin{aligned}
e_{\mathrm{Sp}}\left(4 x x^{\prime}\right) & =4 e_{\mathrm{Sp}}\left(x x^{\prime}\right), \\
S_{t}\left(E_{4}\left(e_{\mathrm{Sp}}\left(4 x x^{\prime}\right)\right)\right) & =S_{t}\left(E_{1}\left(e_{\mathrm{Sp}}\left(x x^{\prime}\right)\right)\right)^{4} \\
& =\left(\left(w_{1}+w_{1}^{\prime}\right)^{4}+t_{1}\left(w_{1}+w_{1}^{\prime}\right)^{8}+\cdots\right)^{4},
\end{aligned}
$$

where $w_{1}$ and $w_{1}^{\prime}$ are the Stiefel-Whitney classes of the canonical bundles over the two factors, and the restriction map has been omitted from the notation. This uses formulas (1) and (2) of the preceding section, the fact about the restriction of $p_{1}^{S}$ of a symplectic line bundle, and the fact that $w_{1}^{\prime}$ is additive over tensor products of line bundles.

This gives, from the equation above, and replacing $S_{t}$ by $S_{t 0}$

$$
\begin{aligned}
\left(\left(w_{1}+w_{1}^{\prime}\right)^{4}+t_{1}^{4}\left(w_{1}\right.\right. & \left.\left.+w_{1}^{\prime}\right)^{8}+\cdots\right)^{4} \\
& =\sum S_{t 0}\left(m_{i j}\right)\left(w_{1}^{4}+w_{1}^{8} t_{1}^{4}+\cdots\right)^{i}\left(w_{1}^{\prime 4}+w_{1}^{\prime 8} t_{1}^{4}+\cdots\right)^{j} .
\end{aligned}
$$

Let $u=g\left(w_{1}^{\prime}\right)$ denote $w_{1}+u_{1}^{2} t_{1}+\cdots$ and $v=g\left(w_{1}^{\prime}\right)$. As a formal power series $g$ is invertible, having leading term $w_{1}$. So the equation above can be written

$$
\left(g\left(g^{-1}(u)+g^{-1}(v)\right)\right)^{16}=\sum S_{t 0}\left(m_{i j}\right) u^{4 i} v^{4 j} .
$$

It can be noted that the left-hand side is the 16 th power of the formal group law of unoriented cobordism theory. However here the theorem will be proved with the background of [7].

It can be observed from the left-hand side that $S_{t 0}\left(m_{i j}\right)$ will be a 16 th power in the ring over $Z_{2}$ generated by the $t$ 's.

This implies that the unoriented cobordism classes of the $m_{i j}$ are 16th powers, since $S_{t 0}$ composed with the ring homomorphism which sends $t_{2^{k}-1}$ to zero for all $k$ and preserves the other $t_{n}$ is an isomorphism from unoriented cobordism to the ring of mod 2 polynomials in the $t_{n}$ other than $t_{2^{k}-1}$. This follows from the lemma on p. 96 of Stong [7] which says that unoriented cobordism is a polynomial algebra on generators of degrees of all positive integers other than those of the form $2^{k}-1$, and that the generator in degree $n$ has a nonzero $s_{(n)}$ number. Therefore $S_{t 0}$ will map it to $t_{n}+$ decomposables, a generator in the image. Next it will be shown that for every $n$ not of the form $2^{k}-1$, there will be some $m_{i j}$ whose $S_{t 0}$ class has a term of the form $t_{n}^{16}$ when expressed in terms of the monomials in the $t$ 's. Then the 16th root of this class will be a generator in dimension $n$, and it will be proved that the $m_{i j}$ generate the ring of 16 th powers.

In order to prove this we will take the ring homomorphism on the ring 
in the $t$ 's which sends every $t_{k}$ other than $t_{n}$ to zero, and apply it to both sides of the equation above. Ring homomorphisms on the coefficients preserve composition and inverses of formal power series.

In the ring,

$$
\begin{aligned}
g(x)= & x+t_{n} x^{n+1}, \\
g^{-1}(x)= & x+t_{n} x^{n+1}+\text { terms of higher degree, } \\
g\left(g^{-1}(a)+g^{-1}(b)\right)= & \left(a+b+t_{n} a^{n+1}+t_{n} b^{n+1}\right)+t_{n}(a+b)^{n+1} \\
& + \text { terms of higher degree. }
\end{aligned}
$$

If $n+1=2^{k}(2 j+1),(a+b)^{n+1}=\left(a^{2^{k}}+b^{2^{k}}\right)^{2 j+1}$ in which the coefficient of $a^{2^{k}} b^{2^{k}(2 j)}$ is nonzero. If $n \neq 2^{k}-1, j$ will be nonzero, and the coefficient of $t_{n}$ in $g\left(g^{-1}(a)+g^{-1}(b)\right)$ will be nonzero. So in the equation on p. 6 the coefficient of $t_{n}^{16}$ will be nonzero. This proves the theorem.

III. Remarks. Floyd's paper [2] leaves open the possibility that certain 8 th powers as well as 16 th powers may be in the image. I have not found any way of constructing such classes. If they do not exist, this would be a counterexample to Ray's conjecture that $K O$-theory decides symplectic cobordism, by considering the square of $\frac{1}{2}$ of one of Stong's manifolds [6] of dimension 24.

In the remainder of this section some alternative constructions for 16 th powers to be symplectic images will be given.

(1) Start with an $H P^{n}$. It has the canonical symplectic line bundle over it. Next take the quaternionic projective space bundle of twice this line bundle. There is a new symplectic line bundle over it, which is the canonical bundle restricted to each fibre. Take the submanifold dual to this line bundle. This gives the manifolds of Landweber [3], which have symplectic stable tangent bundles, but map to zero in unoriented cobordism.

Over these manifolds one has the restriction of the second symplectic line bundles. Take the quaternionic projective space bundles of $4 k$ times these restricted bundles + a trivial line bundle. There is a third symplectic line bundle which is the canonical bundle restricted to each fibre of this bundle. Take the quaternionic projective space bundle of twice this symplectic line bundle, which has a 4th symplectic line bundle constructed like the others over it. Finally take the submanifold dual to the 4th line bundle. These manifolds are always stably almost symplectic, and for suitable $n$ and $k$ are unoriented representatives of 16th powers of generators.

The proof of this paper suggests that another construction might be to take a product of Landweber's manifolds and then dualize the symplectification of the tensor product of the symplectic line bundles (the ones above) over them. 
A final alternative is that Landweber's manifolds can be replaced by others where one uses $\left(S^{2} \times C P^{4 n+1}\right) / Z_{2}$ in place of the second stage of their construction. Here $Z_{2}$ acts on $S^{2}$ in $R^{3}$ by $(x, y, z)$ goes to $(-x, y, z)$ and on $C P^{4 n+1}$ by the action of the quaternion $j$ (the quotient by the nonzero complex numbers of its action on $H^{2 n+1}$ ).

\section{BIBLIOGRAPHY}

1. P. E. Conner and E. E. Floyd, The relation of cobordism to $K$-theories, Lecture Notes in Math., no. 28, Springer-Verlag, Berlin and New York, 1966. MR 35 \#7344.

2. E. E. Floyd, Stiefel-Whitney numbers of quaternionic and related manifolds, Trans. Amer. Math. Soc. 155 (1971), 77-94.

3. Peter S. Landweber, On the symplectic bordism groups of the spaces $\operatorname{Sp}(n), \operatorname{HP}(n)$, and $\operatorname{BSp}(n)$, Michigan Math. J. 15 (1968), 145-153. MR 37 \#2237.

4. Nigel Ray, $A$ note on the symplectic bordism ring, Bull. London Math. Soc. 3 (1971), 159-162.

5. David M. Segal, Symplectic cobordism of manifolds, Comment. Math. Helv. 45 (1970), 159-169.

6. R. E. Stong, Some remarks on symplectic cobordism, Ann. of Math. (2) 86 (1967), 425-433. MR 36 \#2162.

7. — Notes on cobordism theory, Math. Notes, Princeton Univ. Press, Princeton, N.J.; Univ. of Tokyo Press, Tokyo, 1968. MR 40 \#2108.

Department of Mathematics, University of Georgia, Athens, Georgia 30601 